\title{
Measurement of Charged Current Triple Gauge Boson Couplings
}

\author{
Stefano Villa* \\ University of California, Riverside, CA 92521, USA \\ E-mail: 'Stefano.Vilia@cern.ch'
}

ABSTRACT: We review the status of measurements of charged current Triple Gauge Couplings based on the study of single and double gauge boson production at LEP. Different methods of extraction are presented, involving the W-pair, single $\mathrm{W}$ and single photon channels. The related topics of spin density matrix and $\mathrm{W}$ polarisation determination are also described.

The non-Abelian gauge structure of the electroweak theory implies the existence of Triple Gauge Couplings (TGC) of the type WWZ and WW $\gamma$, between the two charged bosons $\mathrm{W}^{+}$and $\mathrm{W}^{-}$and one of the neutral bosons $\mathrm{Z}$ or $\gamma$. The measurement of the strength of such couplings is not only a way of directly testing the gauge structure of the theory, but also a powerful tool for setting limits on possible deviations from it.

For this purpose the most general Lorentz invariant Lagrangian of the type WWV (where $\mathrm{V}$ can be either $\mathrm{Z}$ or $\gamma$ ) is written, making use of the gauge boson fields and of their covariant derivatives [i] be necessary to parametrise such interactions: $g_{1}^{V}, \kappa_{V}, \lambda_{V}, g_{5}^{V}, g_{4}^{V}, \tilde{\kappa}_{V}$ and $\tilde{\lambda}_{V}$, with $\mathrm{V}=\mathrm{Z}$ or $\gamma$. A reduction of the set of couplings being measured is usually obtained by assuming the conservation of well established symmetries also in the case of anomalous (i.e. different from the Standard Model ones) couplings.

The assumption of electromagnetic gauge invariance and of conservation of $\mathrm{C}$ and $\mathrm{P}$ symmetries leads to a reduction of the number of parameters to $5: g_{1}^{Z}, \kappa_{\gamma}, \kappa_{Z}, \lambda_{\gamma}$ and $\lambda_{Z}$. A further reduction is achieved by assuming the custodial SU(2) symmetry, which

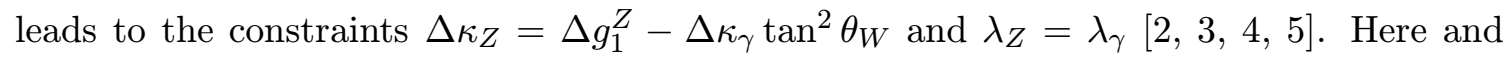
in the following $\Delta$ denotes the deviation from the Standard Model value of the couplings: $\Delta g_{1}^{Z} \equiv g_{1}^{Z}-1, \Delta \kappa_{\gamma} \equiv \kappa_{\gamma}-1$ and $\Delta \kappa_{Z} \equiv \kappa_{Z}-1$.

This paper is organised as follows: Sec. 1י1-1 describes the methods of TGC measurements based on WW production at LEP, Sec.

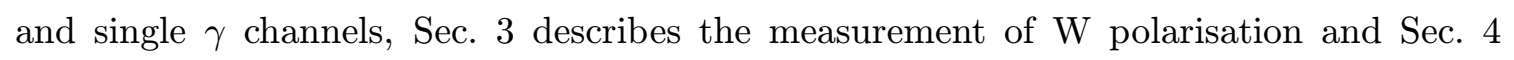
contains some conclusions. 


\section{Measurement of TGC's from WW production}

Anomalous Couplings would affect polarization, production angles and total cross section of WW. The kinematics of a WW event is described with 5 variables (neglecting $\gamma$ radiation and fixing the $\mathrm{W}$ mass): the polar angle of the $W^{-}$, the polar and azimuthal angles of the fermion in the $W^{-}$rest frame and the polar and azimuthal angles of the antifermion in the $W^{+}$rest frame. The $\mathrm{W}$ pair decays with $45.6 \%$ probability into 4 jets (qqqq), with $43.8 \%$ probability into 2 jets and a charged lepton and neutrino pair ( $q q \mathrm{e} \nu, q q \mu \nu$ or $q q \tau \nu)$ and in $10.6 \%$ of the cases in two lepton-neutrino pairs $(\ell \nu \ell \nu)$. These channels are normally treated separately for the purpose of TGC extraction, due to the different amount of information which is lost with neutrino emission and to the impossibility of reconstructing the charge of the primary quarks from the hadronic jets.

The high accuracy reached by $\mathrm{W}$ physics at LEP has stimulated a theoretical effort to improve the description of WW production. New Monte Carlo (MC) generators have

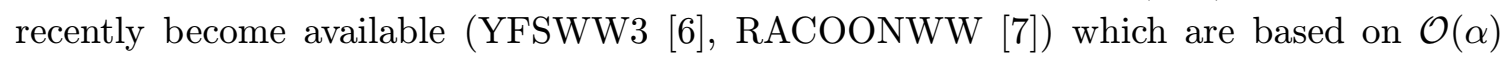
description of WW production in the framework of Double Pole Approximation. With respect to the previously available and less accurate generators the $\mathcal{O}(\alpha)$ MC's predict a lower WW cross section (of about $2.5 \%$ at $200 \mathrm{GeVcentre-of-mass} \mathrm{energy),} \mathrm{and} \mathrm{a} \mathrm{sizeable}$ (about $2 \%$ ) change in the slope of the $\cos \theta_{W^{-}}$distribution. The implementation and testing of the new generators is a non-trivial task and LEP experiments are still working on it, with the common goal of using the new generators for the final LEP publications on W physics; the only preliminary results which take into account $\mathcal{O}(\alpha)$ corrections have been published by ALEPH; all other LEP experiments have not released any results.

Different strategies are used at LEP to extract the TGC's from WW events; we will shortly describe them in the following.

\subsection{Fit of the multidimensional distribution of phase-space angles}

The L3 analysis [i్⿴囗十)] is based on a binned maximum likelihood fit in the 5D phase space of the production and decay angles described above. In the different decay channels of WW production some ambiguity arises due to the unknown quark flavour; the angular distributions of the hadronic decay products are therefore correspondingly folded. The Monte Carlo is generated with the Standard Model set of couplings and the effect of anomalous couplings is obtained through event-by-event reweighting. A somehow similar method is the one used by ALEPH [ $\left[\bar{g}_{1}^{1},{ }_{1}^{1} \bar{O}^{1}\right]$ in their measurement of real and imaginary part of CP violating couplings. An unbinned maximum likelihood fit is performed, based on probability density functions obtained convoluting the lowest order narrow-width differential cross section with detector resolution and efficiency and initial state radiation function.

\subsection{Optimal Observables}

The method of Optimal Observables (OO) projects the information which is contained in the phase space angles $(\Omega)$ into some newly defined variables, called optimal observables. 
Since the Lagrangian is linear in the couplings $(\alpha)$, the cross section is a quadratic function:

$$
\frac{\mathrm{d} \sigma(\alpha)}{\mathrm{d} \Omega}=c_{0}(\Omega)+\sum_{i} c_{1}^{i}(\Omega) \alpha_{i}+\sum_{i \leq j} c_{2}^{i j}(\Omega) \alpha_{i} \alpha_{j}
$$

The optimal observables are defined as $\mathcal{O}_{i}^{1}=\frac{c_{1}^{i}(\Omega)}{c_{0}(\Omega)}, \mathcal{O}_{i j}^{2}=\frac{c_{2}^{i j}(\Omega)}{c_{0}(\Omega)}$ and they contain the same information as the phase-space angles in a reduced set of variables. The fit of more than one coupling would nevertheless require the introduction of more and more variables, somehow spoiling the usefulness of the method. Some approximations can reduce the number of variables: for small $\alpha$, one can neglect the second order OO's, and also assume that all the information is contained in the mean of the $\mathrm{OO}$ rather than in the full distribution.

\begin{tabular}{|c|c|c|}
\hline Coupling & Fit result & $95 \%$ CL \\
\hline$\Delta g_{1}^{Z}$ & $0.015_{-0.032}^{+0.035}$ & {$[-0.048,0.080]$} \\
$\Delta \kappa_{\gamma}$ & $-0.021_{-0.073}^{+0.073}$ & {$[-0.164,0.134]$} \\
$\lambda_{\gamma}$ & $-0.001_{-0.031}^{+0.034}$ & {$[-0.059,0.065]$} \\
\hline
\end{tabular}

Table 1: Preliminary results of the ALEPH measurement of $\Delta g_{1}^{Z}, \Delta \kappa_{\gamma}$ and $\lambda_{\gamma}$ based on WW, single $\mathrm{W}$ and single $\gamma$ events collected at centre-of-mass energies between $172 \mathrm{GeV}$ and $208 \mathrm{GeV}$ [i 10.01 .
The fit of mean OO is used by both

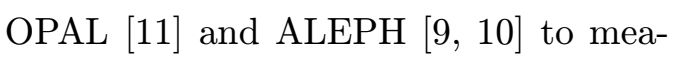
sure TGC's, by minimisation of a $\chi^{2}$ built out of the expected and measured mean values of the first and second order optimal observables. The preliminary results of the ALEPH fit, based on data collected at centre-of-mass energies between $172 \mathrm{GeV}$ and $208 \mathrm{GeV}$, combined

with single $\mathrm{W}$ and single $\gamma$ results, are reported in table

The DELPHI analysis of the $q q q q$ channel with 2 non-zero couplings [1] $[1]$ is based on an iterative procedure. A 2D binned fit of the distribution of the first order Optimal Observables of the two couplings is performed and the procedure is repeated each time expanding the differential cross section about the values of the couplings obtained in the previous iteration. Convergence is usually achieved after 3 or 4 iterations.

\subsection{Spin Density Matrix analysis}

The Spin Density Matrix (SDM) is a physical quantity which allows the study of TGC's in a model independent way, without the need of a specific parametrisation. The SDM for $\mathrm{W}$ pair production is defined as:

$$
\rho_{\tau_{-} \tau_{-}^{\prime} \tau_{+} \tau_{+}^{\prime}}\left(s, \cos \theta_{W}\right)=\frac{\sum_{\lambda} F_{\tau_{-} \tau_{+}}^{\lambda}\left(F_{\tau_{-}^{\prime} \tau_{+}^{\prime}}^{\lambda}\right)^{*}}{\sum_{\lambda \tau_{-} \tau_{+}}\left|F_{\tau_{-} \tau_{+}}^{\lambda}\right|^{2}},
$$

where $F_{\tau_{-} \tau_{+}}^{\lambda}$ is the amplitude for producing a $\mathrm{W}^{+}$with helicity $\tau^{+}$and a $\mathrm{W}^{-}$with helicity $\tau^{-}$when $\mathrm{e}^{-}$has helicity $\lambda$. The SDM has 80 independent real coefficients; for experimental purposes it is therefore convenient to introduce the single particle density matrix:

$$
\rho_{\tau_{-} \tau_{-}^{\prime}}^{W^{-}}\left(s, \cos \theta_{W}\right)=\sum_{\tau_{+}} \rho_{\tau_{-} \tau_{-}^{\prime} \tau_{+} \tau_{+}}\left(s, \cos \theta_{W}\right) .
$$


$\mathrm{CP}$ and CPT invariance imply that all elements of $\rho_{\tau_{1} \tau_{2}}^{W}$ are real. Thus, the imaginary parts of the single particle density matrix are sensitive to the CP-violating couplings. SDM elements can be obtained from angular differential cross sections by application of projection operators. Analysis of SDM have been performed in DELPHI [1] [1] from the OPAL analysis are shown in table $\bar{z}$.

$\begin{array}{lll}\tilde{\kappa}_{Z}= & -0.20_{-0.07}^{+0.10} \\ g_{4}^{Z} & =-0.02_{-0.33}^{+0.32} \\ \tilde{\lambda}_{Z} & =-0.18_{-0.16}^{+0.24}\end{array}$

Table 2: OPAL measurement of CP-violating couplings using SDM analysis, based on data collected at $\sqrt{s}=189 \mathrm{GeV}$.

\section{TGC's from single $\mathbf{W}$ and single $\gamma$}

\begin{tabular}{|lr|c|c|}
\hline & & $\lambda_{\gamma}$ & $\Delta \kappa_{\gamma}$ \\
\hline ALEPH & $161-202 \mathrm{GeV}$ & {$[-0.57,0.44] 95 \% \mathrm{CL}$} & {$[-0.54,0.15] 95 \% \mathrm{CL}$} \\
DELPHI & $189 \mathrm{GeV}$ : lept. & $0.48_{-1.29}^{+0.39}$ & $0.23_{-0.39}^{+0.33}$ \\
& hadr. & $0.42_{-1.21}^{+0.39}$ & $0.19_{-0.58}^{+0.36}$ \\
L3 & $161-202 \mathrm{GeV}$ & $-0.20_{-0.19}^{+0.60}$ & $0.10_{-0.13}^{+0.13}$ \\
OPAL & $189 \mathrm{GeV}$ & $-0.44_{-0.24}^{+0.43}$ & $0.06_{-0.19}^{+0.17}$ \\
\hline
\end{tabular}

Table 3: TGC measurements based on Single W at LEP. The energy range considered in each analysis is specified in the first column.

The single $\mathrm{W}$ process is characterised by the production of the final state electron with very low polar angle, such that it escapes detection. Only the decay products of a single $\mathrm{W}$ boson are observed. This process is sensitive to $\mathrm{WW} \gamma$ couplings and its study can complement the WW channel study in particular for the measurement of $\Delta \kappa_{\gamma}$. TGC extraction is normally based on fits of total cross section, lepton energy spectrum and production angle, and $\mathrm{P}_{t}$ of the jet pair for hadronic single W's. Table $\overline{3}$ summarises TGC mesurements at LEP based on Single W production [i] $\left.{ }_{1}^{1} \bar{j}_{1}\right]$.

Single photon production is sensitive to $\Delta \kappa_{\gamma}$ and $\lambda_{\gamma}$ through the W-W fusion diagram; the most sensitive variables are the total cross section and the energy and production angle

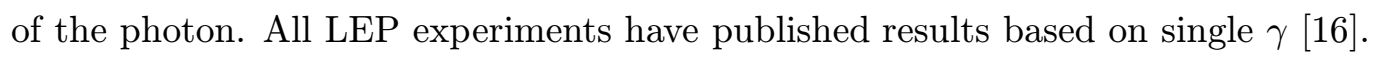

\section{W polarisation}

The study of $\mathrm{W}$ polarisation constitutes a modelindependent way of testing the gauge structure of the electro-weak theory and is therefore complementary to the direct measurement of gauge couplings. Different $\mathrm{W}^{ \pm}$helicity states result in different angular distributions of $\mathrm{W}$ decay products; one can therefore parametrise and fit the leptonic and hadronic decay angle distributions to extract the helicity fractions. Results from the L3 study [i] $\left.\overline{1}_{1}\right]$

\begin{tabular}{|c|c|c|}
\hline helicity & Data & MC \\
\hline-1 & $0.647 \pm 0.066$ & 0.623 \\
+1 & $0.137 \pm 0.034$ & 0.157 \\
0 & $0.216 \pm 0.053$ & 0.220 \\
\hline
\end{tabular}

Table 4: W helicity fractions as measured by L3 using WW events collected at an average $\sqrt{s}$ of $206.6 \mathrm{GeV}$. MC predictions are also shown. are reported in table 
of the correlations of the spins of pair produced $\mathrm{W}^{+}$and $\mathrm{W}^{-}$; good agreement between data and MC is observed. WW spin correlations are observed with a $3.6 \sigma$ significance.

\section{Conclusion}

The status of the measurement of charged triple gauge couplings at LEP has been reviewed. All LEP experiments are in the process of implementing and testing the new $\mathcal{O}(\alpha) \mathrm{MC}$ generators; only ALEPH has published preliminary results based on an implementation of the $\mathcal{O}(\alpha)$ corrections via reweighting of KoralW events, while the other experiments have not released any results. We have therefore given an overview of the methods which are used by LEP experiments to extract TGC measurements from WW, single W and single $\gamma$ production. The measurement of $\mathrm{W}$ polarisation has also been described. All results shown are in good agreement with the Standard Model expectations.

\section{References}

[1] K. Hagiwara, R.D. Peccei, D. Zeppenfeld and K. Hikasa,

[2] G. Gounaris et al., in Physics at LEP 2, Report CERN 96-01 (1996), eds G. Altarelli, T. Sjöstrand, F. Zwirner, Vol. 1, p. 525.

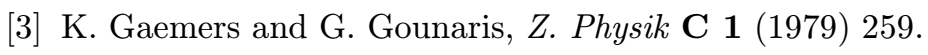

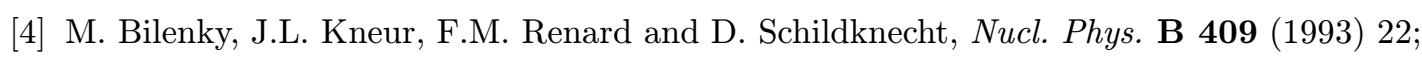
iNucl. Phys. B $4199(1994) 240$

[5] I. Kuss and D. Schildknecht, 'Phys. Lett. B $\mathbf{3} 8 \mathbf{8} \mathbf{3}(1996) 470$

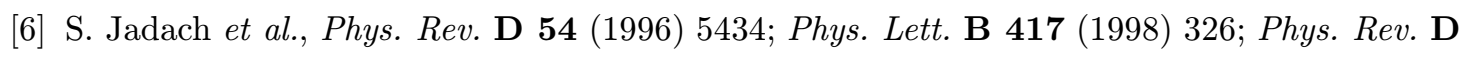
6

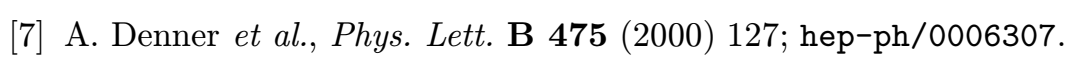

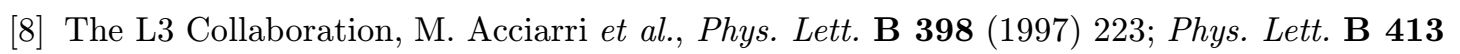
(1997) 176; Phys. Lett. B 467 (1999) $171 ;$ L3 Note 2567 (2000).

[9] The ALEPH Collaboration, R. Barate et al., 'P

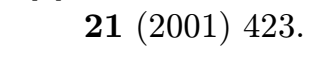

[10] ALEPH note 2001-060, contributed paper to EPS HEP 2001.

[11] The OPAL Collaboration, K. Ackerstaff et al., 'Phys. Lett. B 397-1997) 1475'Eur. Phys. J.' $\overline{\mathbf{C}}_{1}$

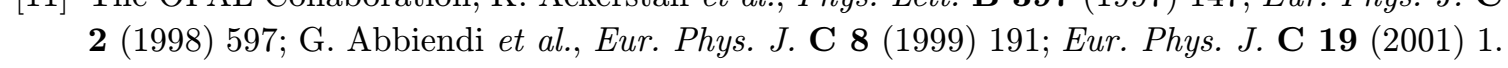

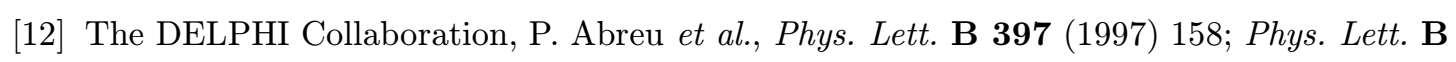
i

[13] DELPHI note 2001-098, contributed paper to EPS HEP 2001.

[14] The OPAL Collaboration, G. Abbiendi et al., 'Eur. Phys. J. C. $\mathbf{1} \overline{\mathbf{9}}(\overline{2} \overline{0} \overline{0} \overline{1}) 229$ '. 


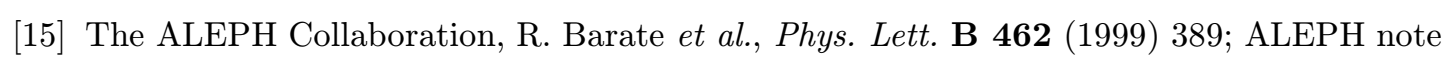
2001-017, contributed paper to EPS HEP 2001.

The DELPHI Collaboration, P. Abreu et al., CERN-EP 2001-033; DELPHI note 2001-099, contributed paper to EPS HEP 2001.

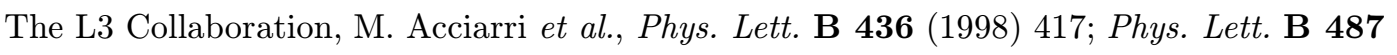

:-

The OPAL Collaboration, G. Abbiendi et al., OPAL preliminary note PN427 (2000).

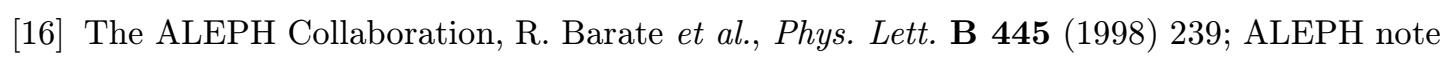
2001-060, contributed paper to EPS HEP 2001.

The DELPHI Collaboration, P. Abreu et al., 'Phys. Lett. B $\mathbf{5 0 2}(200 \overline{1})_{1}^{\prime}$

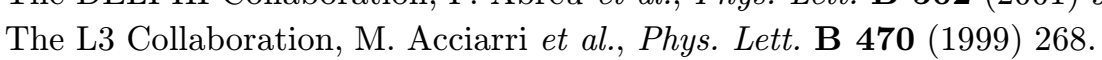

The OPAL Collaboration, G. Abbiendi et al., OPPĀL preliminary note PN481 (2001), contributed paper to EPS HEP 2001.

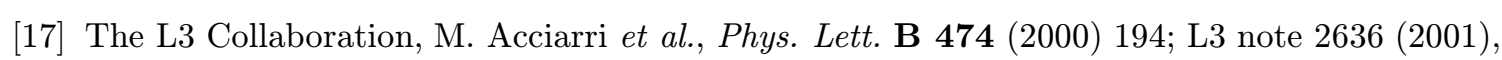
contributed paper to EPS HEP 2001. 